\title{
The Multi-commodity Capacitated Multi-facility Weber Problem: Heuristics and Confidence Intervals
}

\author{
M. Hakan Akyüz*†, Temel Öncan $\ddagger$ İ. Kuban Altınel ${ }^{\S}$
}

\begin{abstract}
The Capacitated Multi-facility Weber Problem is concerned with locating $I$ capacitated facilities in the plane to satisfy the demand of $J$ customers with the minimum total transportation cost of a single commodity. This is a non-convex optimization problem and difficult to solve. In this work, we focus on a multi-commodity extension and consider the situation where $K$ distinct commodities are shipped to the customers subject to capacity and demand constraints. Customer locations, demands and capacities for each commodity are known a priori. The transportation costs, which are proportional to the distance between customers and facilities, depend on the commodity type. We first present a mathematical programming formulation of the problem. Then we propose an alternate location-allocation heuristic and a discrete approximation method which are used to statistically estimate confidence intervals on the optimal objective values. Computational experiments on randomly generated test instances are also included.
\end{abstract}

Keywords: Location-Allocation, Multi-commodity Transportation, Heuristics, Confidence intervals

\section{Introduction}

Given the locations of $J$ customers and their demands, the Multi-facility Weber Problem (MWP) is concerned with locating $I$ uncapacitated facilities in the plane and allocating them to the customers in order to satisfy their demand at minimum total cost. The objective function of the MWP is neither convex nor concave [7], which makes it hard to solve exactly. It becomes the so-called (single-facility) Weber problem when the objective is to

${ }^{*}$ This work was supported in part by TUBITAK with Grant 107M462 and by Galatasaray University Research Foundation with Grant 07.402.014.

${ }^{\dagger}$ M. H. Akyüz is with the Department of Industrial Engineering, Galatasaray University, Ortaköy, İstanbul, 34357, TÜRKIYE (phone: 90-212-227-4480; fax: 90-212-259-5557; email: mhakyuz@gsu.edu.tr)

$\ddagger_{T}$. Öncan is with the Department of Industrial Engineering, Galatasaray University, Ortaköy, İstanbul, 34357, TÜRKIYYE (email: ytoncan@gsu.edu.tr)

$\S \dot{I}$. K. Altinel is with the Department of Industrial Engineering, Boğaziçi University, Bebek, İstanbul, 34342, TÜRKIYE (email: altinel@boun.edu.tr) determine optimal location of a single facility. In some situations, facilities can have capacity constraints, which gives rise to the Capacitated Multi-facility Weber Problem (CMWP). As can be observed, in an optimal solution of the uncapacitated problem each customer is served from the nearest facility, which is not true for the more restricted CMWP because of the capacity constraints. The demand of a customer can be satisfied from different facilities. In other words, the CMWP is a multi-source problem and belongs to a class of difficult problems. Sherali and Nordai [18] have shown that it is NP-hard even if all customers are located on a straight line. In this work we consider the multi-commodity extension of the CMWP where facilities and customers can send and receive more than one commodity. This problem is concerned with locating $I$ capacitated facilities in the plane in order to satisfy the demand of $J$ customers for $K$ commodities optimally and we call it the Multi-commodity Capacitated Multi-facility Weber Problem (MCMWP) in the sequel.

The CMWP has received considerable research interest starting from the seminal work by Cooper [7] for the Euclidean distance CMWP (ECMWP). Sherali et al.'s branch-and-bound algorithms based on a partitioning of the allocation space for ECMWP and the $l_{r}$-distance CMWP (LCMWP) [17], using the Reformulation Linearization Technique (RLT) [16] are examples for exact solution procedures. They suffer from the inefficiency with increasing problem size, which motivates research for efficient and accurate heuristics. Cooper's early alternating location-transportation heuristic [7] and Aras et al.'s discrete approximation strategy [1] are the earliest and most recent examples. They are both inspired from previous works on the (uncapacitated) MWP: Cooper's celebrated alternate location-allocation (ALA) heuristic [6] and Hansen et al.'s p-Median heuristic [11]. The work by Brandeau and Chiu [4] is somewhat different although it is also on the MWP and inspired from Cooper's ALA heuristic. The authors have employed ALA to create a random sample which is then used to statistically estimate confidence intervals for the optimal objective value.

In this work, we follow this line of research: we first propose two approximate solution procedures which can be randomized through their initial conditions. One of 
them is the Multi-commodity and Capacitated version of ALA (MCALA) and alternately solves location and Multi-commodity Transportation Problems (TPs) after being initialized at random facility locations. The other one generalizes Aras et al.'s discrete approximation strategy [1] proposed for the LCMWP; it is essentially based on the solution of an approximating Mixed-Integer Linear Programming (MILP) problem formulated using a large candidate location set within the convex hull of the customer locations. These approximate solution procedures are then combined with statistical methods to estimate confidence intervals for the optimal objective value of the MCMWP.

The rest of this paper is organized as follows. We give the formulation of the MCMWP and develop two approximate solution procedures, in the next section. This is followed by Section 3 where we present the statistical procedures for estimating confidence intervals on the optimal objective values of Combinatorial Optimization Problems (COPs). In Section 4, we combine them with the new heuristics to statistically estimate confidence intervals on the optimal objective values of the MCMWP. Finally, Section 5 concludes the paper.

\section{The Multi-commodity Capacitated Multi-facility Weber Problem}

\subsection{A Mathematical Programming Formu- lation}

A mathematical programming formulation of the MCMWP can be stated as

MCMWP:

$$
\begin{array}{ll}
\min z= & \sum_{i=1}^{I} \sum_{j=1}^{J} \sum_{k=1}^{K} w_{i j k} c_{i j k} d\left(x_{i}, a_{j}\right) \\
\text { s.t. } \quad & \sum_{j=1}^{J} w_{i j k}=s_{i k}, \quad i=1, \ldots, I ; k=1, \ldots, K \\
& \sum_{i=1}^{I} w_{i j k}=q_{j k}, \quad j=1, \ldots, J ; k=1, \ldots, K \\
& \sum_{k=1}^{K} w_{i j k} \leq u_{i j}, \quad i=1, \ldots, I ; j=1, \ldots, J \\
& w_{i j k} \geq 0, \quad \begin{array}{l}
i=1, \ldots, I ; j=1, \ldots, J \\
k=1, \ldots, K .
\end{array}
\end{array}
$$

Here, $J$ is the number of customers, $I$ is the number of facilities to be located and $K$ denotes the number of commodities. $\mathbf{a}_{j}=\left(a_{j 1}, a_{j 2}\right)^{T}$ and $q_{j k}$ represent the coordinates and its demand for commodity $k$ of customer $j$, respectively. The capacity of facility $i$ for commodity $k$ is given by $s_{i k}$ and $\mathbf{x}_{i}=\left(x_{i 1}, x_{i 2}\right)^{T}$ denotes its unknown coordinates. The allocations $w_{i j k}$ are also unknown and represent the amount of commodity $k$ to be shipped from facility $i$ to customer $j$ with the unit shipment cost per unit distance being $c_{i j k}$. We assume that, according to regulations, total amount of allocations on a road connecting facility $i$ with customer $j$ can not be larger than the given upper bound $u_{i j}$. This is formulated with the road capacity constraints (4). The other two sets are the demand and resource constraints. This formulation assumes that the problem is balanced, i.e., the total demand is equal to the total capacity of the facilities for each commodity.

It can be easily shown that an optimal solution of the MCMWP always occurs at an extreme point of the polyhedron defined by (2)-(5), independent of the type of the distance function $d\left(x_{i}, a_{j}\right)$, which measures the distance between facility $i$ and customer $j$. Starting with the seminal work by Cooper [7] different distance functions have been used: the Euclidean, squared Euclidean, rectilinear, and $\ell_{r}$ distances. In this paper we focus on the most general case, the $\ell_{r}$-distance MCMWP with $1 \leq r \leq 2$.

\section{$2.2 \quad$ Approximate Solution Methods}

When the upper bounds $u_{i j}$ are arbitrarily large and $K=$ 1 , namely there is only one commodity, the MCMWP reduces to the CMWP which is known to be very difficult to solve exactly. Hence, the more general MCMWP should be expected to be even more difficult. For that reason, one way to deal with the MCMWP may be the use of the heuristic algorithms. Hence, we propose two such procedures in the following.

\subsubsection{Multi-commodity Capacitated Alternate Location Allocation (MCALA) Heuristic}

Once a feasible transportation plan is given, the MCMWP reduces to solving $I$ Weber problems

$$
\min _{\mathbf{x}_{i}} z=\sum_{j=1}^{J} c_{i j k}^{\prime} d\left(x_{i}, a_{j}\right),
$$

for each facility $i=1, \ldots, I$, each of which can be solved by Weiszfeld's algorithm [19] and its generalizations [5]. Here, $c_{i j k}^{\prime}=\sum_{k=1}^{K} w_{i j k} c_{i j k}$. Additionally, when the facility locations are known, the MCMWP reduces to the solution of the Multi-commodity TP. Consequently, it is possible to tailor Cooper's ALA heuristic [6] to produce a good feasible solution for the MCMWP.

In their work on the dominance and convexity in location theory, Hansen et al. [12] have shown that for the $l_{r}$-distance location problems with $1 \leq r \leq 2$, optimal facility locations lie in the convex hull of customers. Therefore, it becomes possible to randomize MCALA heuristic 
by randomly choosing initial facility locations within the convex hull of customer locations.

\subsubsection{Discrete Approximation (DA)}

The set of optimal locations for the facilities lies in the convex hull of the set of customer locations for the Rectilinear CMWP (RCMWP), Squared Euclidean CMWP (SECMWP), ECMWP and LCMWP with $1 \leq r \leq 2$ [12]. Furthermore, the RCMWP has always an optimal solution with facilities located at the intersection points of vertical and horizontal lines drawn through the customer locations [12]. In their earlier work, Aras et al. [2] have used this property to devise an equivalent MILP formulation of the RCMWP. Although this property is not shared by the ECMWP, SECMWP, and LCMWP, an approximate solution can still be obtained by solving an approximating MILP formulation, which uses an arbitrary number of points in the convex hull of the customer locations representing candidate facility locations [1]. A similar approximating MILP formulation is obtained for the MCMWP which we refer it as the AMCMWP.

The solution of the AMCMWP does not guarantee an optimal solution for the MCMWP unless we are extremely lucky in identifying a set of discrete points in the convex hull of customer locations including optimal facility sites. When the number of candidate points goes to infinity, we would obtain an optimal solution of the original MCMWP. However, there is a trade-off between the quality of the solution provided by the AMCMWP and the required computational effort. It is possible to randomize AMCMWP by randomly selecting the candidate points in the convex hull of customer locations. Furthermore as the final step of the discrete approximation procedure, the MCALA is run as a subprocedure immediately after solving the AMCMWP in order to improve the current solution.

\section{Estimating Statistical Bounds on Op- timal Objective Values}

Heuristics are systematic procedures that compute solutions of optimization problems, which mostly depend on the starting conditions. Hence, it becomes possible to generate a random sample of objective values by running a randomly initialized heuristic. Presumably, objective values are independent of each other and distributed according to the same probability distribution. Then, an immediate question is how to take advantage of this random sample of objective values to estimate the optimal objective value $z^{*}$ of the problem.

\subsection{Point and Interval Estimators}

One possibility is to use Extreme Value Theory (EVT) and benefit from Fisher and Tippett's theorem [8]. According to this theorem, consider $N$ independent samples, each of size $m$, obtained from the same continuous distribution bounded from below (above) by $a$. Let $X_{i}$ denote the minimum (maximum) value of sample $i$, then for $m$ is large enough, $X_{i}$ are Weibull distributed with location parameter $a$. Recall that the probability density and probability distribution functions of the Weibull distribution are respectively

$$
\begin{aligned}
& f(z)=\left(\frac{c}{b^{c}}\right)(z-a)^{c-1} e^{\left[-\left(\frac{z-a}{b}\right)^{c}\right]} \\
& z \geq a>0, b>0, c>0
\end{aligned}
$$

and

$$
F(z)=1-e^{\left[-\left(\frac{z-a}{b}\right)^{c}\right]},
$$

where $a, b$ and $c$ denote the location, scale and shape parameters, respectively.

Note that the location parameter of the Weibull distribution gives the minimum value of the distribution. The Fisher and Tippett's theorem is valid for any continuous distribution from which the sampling is performed. Hence, it is possible to treat an objective value obtained by a randomly initialized run of the heuristic as the minimum of a large random sample and claim that the distribution of the objective values calculated by the heuristic is approximately Weibull. Then, any point estimate of the location parameter of the Weibull distribution estimated using these heuristic objective values yields a point estimate on the minimum objective value. Moreover, the bounds of any interval estimate of the location parameter give a lower and an upper bound for the optimal objective value of the problem with certain confidence level.

Several researchers have employed this result to provide point and interval estimators of $z^{*}$ for various difficult COPs. Based on the early study of McRoberts [15], the first systematic procedure of the point estimation using EVT is proposed by Golden [9] for the famous Traveling Salesman Problem (TSP). This procedure is later improved by Golden and Alt [10] to compute confidence intervals for the optimal value of large COPs. The authors have defined $z_{l b}=\min \left\{z_{i}: 1 \leq i \leq N\right\}$, where $z_{i}$ is the minimum objective value in sample $i$, and have shown that

$$
\operatorname{Pr}\left\{z_{l b}-b \leq a \leq z_{l b}\right\}=1-e^{-N}
$$

which in fact means that $\left[z_{l b}-b, z_{l b}\right]$ is a $100\left(1-e^{-N}\right) \%$ confidence interval for the location parameter $a$, namely $z^{*}$. Notice that the confidence level is almost 1 even for small values of $N$. Los and Lardinois [14] have suggested to use a subset of size $N^{\prime} \leq N$ local optima with distinct values $z_{1}, z_{2}, \ldots, z_{N^{\prime}}$ to fit a Weibull distribution. 
The reason for this suggestion is that the Fisher and Tippett's theorem assumes the independence of $N$ samples; nevertheless having identical local optima in the set of $N$ samples is equivalent to repeating the same sample several times. It should be noted that Golden and Alt [10] considered a local optimal solution to be the extreme values of a sample when they apply a randomly initialized heuristic and treated the intermediate heuristic step solutions to constitute the corresponding sample. According to Los and Lardinois [14] those samples can have different sizes because the number of intermediate heuristic steps can be different until the convergence of the heuristic. Therefore, the authors have suggested to take $N^{\prime}$ samples each having $m$ distinct local optima and developed the formula

$$
P\left(z_{l b}-\frac{b}{T} \leq a \leq z_{l b}\right)=1-\exp \left(-\frac{N^{\prime}}{T^{c}}\right),
$$

where $T$ is any real number. The main advantage of this formula over (9) is its explicit dependence on the confidence level. In other words, $100(1-\alpha) \%$ confidence interval $\left[z_{l b}-\frac{b}{T}, z_{l b}\right]$ for the location parameter $a$ can be achieved by letting $T=\left(-\frac{N^{\prime}}{\ln \alpha}\right)^{\frac{1}{c}}$. However, there is a specific problem with the Los and Lardinois formula (10): it involves the shape parameter $c$, which can make the confidence intervals wider or narrower than it should be due to the direct dependence on $c$. As a remedy, one can consider to take samples of equal size and apply Golden and Alt's procedure in order to avoid from direct dependence of the confidence interval on the Weibull shape parameter.

\subsection{Procedures to Estimate Weibull Para- meters}

The estimation of the Weibull parameters is a critical issue in the application of the EVT. Basically, three type of estimators are used: Least Square Estimators (LSEs), Simple Point Estimators (SPEs) and Maximum Likelihood Estimators (MLEs).

\subsubsection{The Least Squares Error Estimators}

Golden [9] has pursued the Mc Roberts' method [15] and proposed a LSE for the location parameter $a$. By taking logarithms of the Weibull distribution function (8) twice Golden [9] has obtained

$$
c \ln (z-a)-c \ln b=\ln [-\ln (1-F(z))] .
$$

Observe that when the location parameter $a$ of the Weibull distribution is fixed then (11) can be considered as the equation of a regression line. Their values can be estimated using the least square analysis. The estimation procedure is repeated for different values of location parameter a until the largest correlation coefficient is obtained. A nonlinear regression version of the LSE is proposed by Wilson et al. [20].

\subsubsection{Simple Point Estimators}

Let $z_{1} \leq z_{2} \leq \ldots \leq z_{N}$ be an ordered sample from a Weibull distribution with unknown location parameter a. The SPE or analytical estimator

$$
\widehat{a}=\frac{z_{1} z_{N}-z_{2}^{2}}{z_{1}+z_{N}-2 z_{2}} .
$$

is proposed by Zanakis [21] for the location parameter. The scale parameter $b$ is set to

$$
\widehat{b}=z_{\lceil 0.63 N\rceil}-\widehat{a}
$$

in almost all studies in the literature where $\widehat{a}$ is an estimate of the location parameter. The shape parameter $c$, is the most important parameter which also affects the estimation of other parameters and in case it is miscalculated the confidence intervals produced can be inefficient. Zanakis [21] have proposed to estimate the shape parameter with

$$
\widehat{c}=\frac{2.989}{\ln \left[\left(z_{k}-\widehat{a}\right) /\left(z_{i}-\widehat{a}_{2}\right)\right]}
$$

where $z_{i}$ and $z_{k}$ are the observations with $i=\lceil 0.16731 N\rceil$ $k=\lceil 0.97366 N\rceil$, respectively. Zanakis [21] has indicated that $\widehat{a}, \widehat{b}$ and $\widehat{c}$ produce the least mean squared errors for the Weibull parameters. All these analytic estimators are frequently used in the initialization of a MLE procedure.

\subsubsection{The Maximum Likelihood Estimators}

Let $z_{1} \leq z_{2} \leq \ldots \leq z_{N}$ be $N$ independent observations obtained from a Weibull distribution. The MLE method aims to estimate the best values of the location parameter $a$, the scale parameter $b$ and the shape parameter $c$. In other words, given the Weibull likelihood function

$$
L(\varphi)=\prod_{i=1}^{N} \frac{c}{b^{c}}\left(z_{i}-a\right)^{c-1} \exp \left(-\left(\frac{z_{i}-a}{b}\right)^{c}\right)
$$

where $\varphi=\left(z_{1}, z_{2}, \ldots, z_{N}, a, b, c\right)$ is a vector consisting of observations and parameters, the MLE method estimates $a, b$ and $c$, by maximizing $L(\varphi)$ such that $a \leq z_{1}, b \geq 0$ and $c \geq 0$. This problem can be solved by using nonlinear optimization techniques. Since the maximization of $L(\varphi)$ is equivalent to the maximization of the log-likelihood $\ln (L(\varphi))$, an approach is the solution of the equality system obtained by setting the partial derivative $\ln (L(\varphi))$ with respect to $a, b$ and $c$, to zero as done by Golden [9]. The MLE procedure requires to find the maximum of a non-convex function and it is not a trivial task. However, Zanakis [21] has stated that in general parameter estimation by the MLE generate sample fits better than the SPEs particularly when the shape parameter becomes larger. 


\section{Computational Experiments}

In this section we present the computational experiments with the MCALA and DA heuristics. They are implemented in $\mathrm{C}^{++}$and run on a Dell Server PE2900 with two $3.16 \mathrm{GHz}$ Quad Core Processors and 16 GB RAM operating within Microsoft Windows Server 2003. Cplex 11.0 with default options is used as a subroutine to solve the Multi-commodity TPs and the AMCMWPs which are part of the MCALA and DA algorithms, respectively.

In the literature, there is no standard test library for the MCMWP and hence we have generated our test instances to carry out computational experiments. For this purpose, 30 Euclidean distance MCMWP (EMCMWP) instances of various sizes have been produced.

We have run each of the MCALA and DA heuristic 20000 times and obtained the benchmark global minimum as the best of 40000 runs. The quality of confidence intervals are evaluated with respect to these benchmark global minimums. The initial facility locations for both heuristics are randomly selected within the convex hull of the customer locations. The MCALA heuristic requires the initialization of $I$ random facility locations while, the DA algorithm requires $3 \times J$ random candidate facility locations.

We have performed two sampling schemes: the McRoberts' Approach (MRA) [15] and the Los and Lardinois' Approach (LLA) [14]. In the MRA, intermediate solutions obtained during the run of our randomly initialized heuristics constitute the samples. As a successful application of the MRA we refer to Golden and Alt [10]. Later on, Los and Lardinois [14] have claimed that the sample generation method of MRA may harm the independence of the sample and they have suggested to use $m \times N$ distinct observations. Indeed, the requirement that all observations should be distinct is not necessary [20]. As a result, the LLA the samples can be constructed of $m$ observations which are not necessarily distinct.

Using both the MRA and LLA, we have generated samples from two parent populations each consisting of previously generated 20000 randomly initialized solutions with the MCALA and DA heuristics. In our MRA implementation, we have considered samples of size $N=20,30$ and 40 each consisting of the intermediate feasible solutions of randomly initialized heuristics (i.e. MCALA and DA). In our LLA implementation, samples of size $N=20,30$ and 40 are taken, where each of them consists of $m=10$ randomly initialized heuristic outputs.

In order to test the independence of the samples we have employed the runs test [3] over the sample minimums with $95 \%$ confidence level. On the instances which have passed the independence test, the K-S test [3] with a $95 \%$ confidence level has been applied to test the hypothesis
Table 1: Summary of the Performance of Statistical Lower Bounding Approach

\begin{tabular}{|c|c|c|c|c|c|}
\hline Heuristic & Method & Size & $\begin{array}{l}\text { Interval } \\
\text { Width } \\
(\%)\end{array}$ & Gap (\%) & $\begin{array}{l}\text { No. Of } \\
\text { Covering } \\
\text { Intervals }\end{array}$ \\
\hline \multirow{3}{*}{ MCALA } & & 20 & 29.3 & 2 & 30 \\
\hline & MRA & 30 & 28.8 & 3.2 & 30 \\
\hline & & 40 & 34.8 & 6.2 & 30 \\
\hline & \multicolumn{2}{|l|}{ Average } & 31 & 3.8 & \\
\hline \multirow{3}{*}{ MCALA } & \multirow{3}{*}{ LLA } & 20 & 21.7 & 1.1 & 26 \\
\hline & & 30 & 21.6 & 1.9 & 26 \\
\hline & & 40 & 23.1 & 2.2 & 23 \\
\hline \multirow{4}{*}{ DA } & Average & & 22.1 & 1.7 & \\
\hline & \multirow{3}{*}{ MRA } & 20 & 21.5 & 1.6 & 27 \\
\hline & & 30 & 22.2 & 1.3 & 25 \\
\hline & & 40 & 24.6 & 2.3 & 24 \\
\hline \multirow{4}{*}{ DA } & Average & & 22.8 & 1.7 & \\
\hline & \multirow{3}{*}{ LLA } & 20 & 25.1 & 1.7 & $20^{*}$ \\
\hline & & 30 & 27.7 & 1.1 & 18 \\
\hline & & 40 & 27.2 & 0.8 & 18 \\
\hline & Average & & 26.7 & 1.2 & \\
\hline
\end{tabular}

that the sample minimums are from a Weibull distribution. In case the sample minimums pass both of these tests, a confidence interval can be determined.

We have obtained the MLEs of the Weibull parameters by maximizing Weibull log-likelihood function $\ln (L(\varphi))$. For that purpose we have employed a modification of the Nelder-Mead simplex search proposed by Lagarias et al. [13]. The initial simplex is constructed by using estimators $\widehat{a}, \widehat{b}$ and $\widehat{c}$ given with (12), (13) and (14), respectively. To test the validity of the confidence intervals, we have implemented the confidence interval procedure proposed by Golden and Alt [10].

In Table 1, a comparison of both sampling approaches and algorithms is summarized. The heuristics used are shown in the first column. The second column stands for the sampling method. The sizes of extreme value samples are presented in the third column. The fourth column denotes the mean confidence interval width which is calculated by taking the average of $100 \times\left(z_{u b}-z_{l b}\right) / z_{l b}$ over all intervals produced. Here, $z_{l b}$ and $z_{u b}$ indicate the lower and upper limits of the corresponding confidence intervals. The fifth column presents the mean absolute gaps between the lower limit of the confidence interval and the benchmark global minimum. Similar to the interval width, they are calculated by taking the average of $100 \times\left|z^{*}-z_{l b}\right| / z^{*}$ over all intervals where $z^{*}$ is the corresponding benchmark global minimum. The average of interval widths and absolute gaps are also presented for each heuristic and sampling method pair. The sixth column indicates the number of instances which have passed the K-S test and the benchmark global minimum lies within the interval. In all but one case, the confidence intervals have covered the benchmark minimum. This case is indicated by an asterisk in Table 1 .

Considering the results given in Table 1, we observe that on the average, the mean confidence interval width and the mean absolute gap obtained with DA algorithm are smaller than the ones produced with the MCALA. On the other hand, the MCALA yields larger number of confidence intervals containing the benchmark minimum than 
the DA algorithm does. We should note that the confidence interval approach applied to the MCMWP instances produces lower bounds within $3.8 \%$ on the average. Hence, we can say that the confidence interval approach using EVT outputs acceptable lower bounds on the objective function values of the MCMWP.

\section{Conclusion}

In this paper, we have addressed the MCMWP which is a generalization of the CMWP. Two approximate solution procedures (i.e. MCALA and DA) have been devised for its solution and have been combined with statistical estimation procedures. Initialized by random starting solutions, the proposed approximate solution procedures are run to produce random objective function values. The Fisher and Tippett's theorem has been applied to produce confidence intervals for the optimal solutions of the randomly generated MCMWP test instances. The initial computational experiments have shown that confidence interval approach employing the EVT has performed well.

\section{References}

[1] Aras, N., Altınel, İ.K., Orbay, M., "New heuristic methods for the capacitated multi-facility Weber problem," Naval Research Logistics, V54, N1, pp. 21-32, 2007.

[2] Aras, N., Orbay, M., Altınel, İ.K., "Efficient heuristics for the rectilinear distance capacitated multifacility Weber problem," Journal of the Operational Research Society, V59,N1, pp. 64-79, 2008.

[3] Beyer, W.H., CRC Handbook of Tables for Probability and Statistics, Second Edition, CRC Press Inc., 1968.

[4] Brandeau, M.L., Chiu, S.S., "Sequential location and allocation: worst case performance and statistical estimation," Location Science, V1, N4, pp. 289 298, 1993.

[5] Brimberg, J., Love, R.F., "Global convergence of a generalized iterative procedure for the minisum location problem with $\mathrm{L}_{p}$ distances," Operations Research, V41, N6, pp. 1153-1163, 1993.

[6] Cooper, L., "Heuristic methods for locationallocation problems," SIAM Review, V6, N1, pp. 3753,1964 .

[7] Cooper, L., "The transportation-location problem," Operations Research, V20, N1, pp. 94-108, 1972.

[8] Fisher, R.A., Tippett, L.H.C., "Limiting forms of the frequency distribution of the largest or smallest member of a sample," Proceedings of the Cambridge Philosophical Society, pp. 180-190 1928.
[9] Golden, B.L., "A statistical approach to the TSP," Networks V7, N3, pp. 209-225, 1977

[10] Golden, B.L., Alt, F.B., "Interval estimation of a global optimum for large combinatorial problems," Naval Research Logistic Quarterly, V26, N1, pp. 6977, 1979.

[11] Hansen, P., Mladenović, N., Taillard, É., "Heuristic solution of the multisource Weber problem as a $p$ median problem," Operations Research Letters, V22, N2-3, pp. 55-62, 1998.

[12] Hansen, P., Perreur, J., Thisse, F., "Location theory, dominance and convexity: Some further results," Operations Research, V28, N5, pp. 1241-1250, 1980.

[13] Lagarias, J.C., Reeds, J.A., Wright, M.H., Wright, P.E., "Convergence properties of the Nelder-Mead simplex algorithm in low dimensions," SIAM Journal on Optimization, V9, N1, pp. 112-147, 1998.

[14] Los, M., Lardinois, C., "Combinatorial programming, statistical optimization and the optimal transportation network problem," Transportation Research B, V16, N2, pp. 89-124, 1982.

[15] McRoberts, K.L., "A search model for evaluating combinatorially explosive problems," Operations Research, V19, N6, pp. 1331-1349, 1971.

[16] Sherali, H.D., Adams, W.P., A reformulationlinearization technique for solving discrete and continuous nonconvex problems, Kluwer Academic Publishers, 1999.

[17] Sherali, H.D., Al-Loughani, I., Subramanian, S., "Global optimization procedures for the capacitated Euclidean and $\mathrm{L}_{p}$ distance multifacility locationallocation problem," Operations Research, V50, N3, pp. 433-448, 2002.

[18] Sherali, H.D., Nordai, F.L., "NP-hard, capacitated, balanced p-median problems on a chain graph with a continuum of link demands," Mathematics of $\mathrm{Op}$ erations Research, V13, N1, pp. 32-49, 1988.

[19] Weiszfeld, E., "Sur le point lequel la somme des distances de $\mathrm{n}$ points donné est minimum," Tôhoku Mathematics Journal, V43, pp. 355-386, 1937.

[20] Wilson, A.D., King, R.E., Wilson, J.R., "Case study on statistically estimating the minimum makespan for flow line scheduling problems," European Journal of Operational Research, V155, N2, pp. 439-454, 2004.

[21] Zanakis, S.H., "Computational experience of some nonlinear optimization algorithms in deriving maximum likelihood estimates for the three-parameter Weibull distribution," TIMS Studies in Management Sciences, V7, pp. 63-77, 1977. 